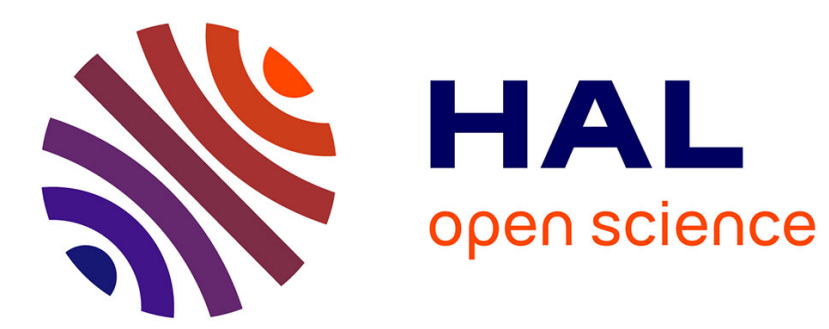

\title{
L'ethnologue sous le regard de deux amis
}

Marc Bordigoni

\section{To cite this version:}

Marc Bordigoni. L'ethnologue sous le regard de deux amis. Etudes Tsiganes, 2012, Hommages, 50

(2012/2), pp.12-17. halshs-01192593

\section{HAL Id: halshs-01192593 \\ https://shs.hal.science/halshs-01192593}

Submitted on 9 Sep 2015

HAL is a multi-disciplinary open access archive for the deposit and dissemination of scientific research documents, whether they are published or not. The documents may come from teaching and research institutions in France or abroad, or from public or private research centers.
L'archive ouverte pluridisciplinaire HAL, est destinée au dépôt et à la diffusion de documents scientifiques de niveau recherche, publiés ou non, émanant des établissements d'enseignement et de recherche français ou étrangers, des laboratoires publics ou privés. 
Marc Bordigoni

\section{L'ethnologue sous le regard de deux amis}

Quand la vie m'a menée à me réintéresser à ce que je nomme la présence tsigane en France et en Europe, après des années d'éloignement du terrain, une des idées évidentes était d'aller voir les « anciens », les chercheurs qui depuis les années soixante avaient publiés des articles et des livres à propos des Tsiganes de France. Philippe de Marne faisait partie de ma liste. Il était d'autant plus intrigant de le rencontrer que je savais qu'il avait peu publié (voir la notice bibliographique) et que dans le même temps les Roms français que je croisais - des Zongrois, ainsi que le monde du Voyage les distinguent et des «tsiganologues », me rapportaient toujours le même propos que je résume sous cette formule réellement entendue : «il sait tout des Roms, leur histoire, les généalogies, et tout et tout, mais il veut plus rien dire ». Je n'ai pas mené mon projet d'aller voir les « anciens ». J'ai rencontré Philippe de Marne une fois, en 2004, quand Roland Cottet préparait son film, «Gitans, Tziganes, Gens du Voyage : le droit de vivre » et que ce fut l'occasion de remettre à Lick le prix Romanès à Cabriès, commune près d'Aix-en-Provence (voir sur le site Idemec la remise du prix). A cette occasion il prit la parole et évoqua son amitié ancienne avec Lick.

Il m'était facile cet été 2013 d'aller rencontrer Lick qui habite toujours le sud de la France ainsi que Grofo, un Rom, qui circule aussi dans la région de Marseille.Ce dernier qu'il avait des points communs avec Philippe de Marne, le même prénom et le fait d'avoir accompli leur service militaire à la même période ; pourtant ils ne sont pas de la même année comme le croit Grofo né en 1942, de Marne est de 1937, mais il a pu, peut-être, retarder son incorporation du fait de la durée deses études supérieures.

Grofo : "Nous sommes allés à l'armée en même temps. Mais lui était de la noblesse, il y avait des militaires dans ses ancêtres alors il est devenu lieutenant très vite, il aurait pu être colonel ou général comme son grand-père, ces gens-là vont vite. »

\section{Une mère aristocratique}

L'origine aristocratique de la famille Lemaire de Marne a marqué ses interlocuteurs :Grofo : «Il vivait dans une forêt (Chaumont sur Taronne en Sologne), il y avait des hectares et des hectares, et ça ne finissait jamais, et il disait «ça c'est à nous » et tu roulais « et ça c'est à nous ». Et il y avait des fermes, deux ou trois. Un jour je suis allé le voir là-bas en Sologne, j'ai demandé ma route dans le pays et on m'a dit vous allez par là-bas, vous passez à côté d'une ferme, c'était une très belle ferme, puis une autre, vide mais bien et après vous verrez une petite maison de briques, c'est là. Sa maison était toute petite, une pièce avec une table et une cheminée et un évier et tous ses livres, et à côté une petite pièce avec son lit. Je lui demandai pourquoi il n'habitait pas la ferme vide qui est belle et grande, il m'a dit qu'il ne pouvait pas la chauffer, cette petite maison il fallait juste quelques bûches pour être bien. Je suis allé là-bas mais nous les Roms on ne restait pas là-bas, on n'aime pas la forêt, il y a des mulé, tu dors et il y a tous les bruits, les chouettes, les grenouilles, les oiseaux, on n'a peur des mulé on ne restait pas. Sa mère était une noble, elle avait un château, elle l'a donné pour faire une maison pour les vieux. Philippe ne parlait pas de sa mère. Elle était supérieure, on ne se 
mélangeait pas, elle aurait sûrement voulu le placer dans la politique, Premier ministre ou quelque chose ... »

Lick : « les Roms ne venaient pas au château. Sa mère ne s'intéressait pas, ne se mélangeait pas. Il m'a dit « les autres châtelains l'invitaient chez eux mais elle les invitait au restaurant parce qu'elle ne veut que personne ne vienne au château. Et moi j'ai eu le privilège de manger au château mais dans la cuisine bien sûre, mais si tu savais comme je m'en foutais. ... Lui c'était un petit garçon devant sa mère je le lui disais d'ailleurs. La preuve c'est que s'il n'était pas à l'heure il dormait dehors. » Lick se souvient d'un jour où étant à Neuilly où Mme de Marne disposait d'un appartement Philippe lui dit qu'il doit aller en Sologne. Comme d'habitude ils conversaient des heures durant. Plusieurs fois Philippe veut partir et explique « si je n'arrive pas à l'heure le portail sera fermé ». Et effectivement Lick reçoit quelques jours plus tard une lettre dans laquelle Philippe lui précise qu'il a trouvé porte close et a dû dormir dans les bois. Philippe était généreux, il a hébergé Lick dans l'appartement de Neuilly mais « il avait très, très peur de sa mère, il était resté petit garçon, il avait très peur que sa mère vienne à Neuilly et qu'elle découvre que je dormais là, que même j'amenais des copines, à l'époque quoi (Lick n'était pas encore marié), mais il avait très peur. »

\section{Un chercheur au CNRS}

Lick avait connu Philippe de Marne au cours d'une réunion aux Études tsiganes. Il était monté à Paris pour tenter sa chance comme auteur-compositeur. Il décrit la scène dans un chapitre intitulé « l'ethnologue et le bohémien »:

Au matin, vers neuf, (je me) trouve dans une salle de réunion des Etudes tsiganes, avec des travailleurs sociaux et des gens bénévoles réunis afin de trouver les meilleures solutionsd'intégration, pour ne pas dire assimilation - au problème des Gens du Voyage. En fin de réunion, un jeune gadjo à l'allure distinguée, avec son manteau pied-de-poule, au profil d'intellectuel avec un nez Louis XVI soutenant bien ses lunettes de vue, est surpris de voir un jeune Tsigane rester assis sagement jusqu'au bout de ce colloque. »

Dans la rue la conversation s'engage entre les deux hommes. Le gadjo s'adresse tzigane en romanes, celui-ci lui répond :

«Je ne suis pas Rom, moi, je suis Sinto..

-Ah, excuse-moi je croyais, fait le jeune homme avec respect, continuant tout de même de lui parler moitié romanès et moitié français.

-Tu sais, je connais bien les Roms, et pas trop les Sinti ; personnellement je suis ethnologue, je travaille au CNRS, au musée de l'homme à Paris. .... ...

-Qu'est-ce que cela veut dire, ethnologue ?

-C'est étudié les traditions et la culture des autres d'autres civilisations. »

(Il était une fois les Bohémiens, p.117-118).

Grofo et Lick se souviennent bien de Philippe de Marne ethnologue au CNRS affecté au Musée des arts et traditions populaires. Il leur a parlé de son séjour en Amazonie, en Guyane plus précisément. Selon Grofo Philippe de Marne a fourni une explication à son retour et au 
fait qu'il n'ait pas rien publié sur son séjour amérindien : « Il m'a dit qu'ils (les Indiens) lui ont mangé son crayon, un crayon noir pas un bic et même son appareil photo, enfin c'est pour ça qu'il n'a rien écrit. Il n'a pas donné de nouvelles, il n'y avait pas de téléphone et il n'avait plus de crayon et le musée a dit qu'il était mort. Et puis il est revenu. » Lick raconte la suite : «Je lui ai demandé - qu'est-ce qui t'a fait venir chez les Tsiganes ?et il m'a dit - quand je suis revenu de Guyane on m'a proposé soit les Arabes soit les Tsiganes, et alors c'est comme ça que j'ai fait connaissance avec les Roms de Romainville. Et puis il est parti en voyage avec les Roms, c'est comme ça qu'il a appris la langue, et tout. »

\section{Les Roms}

Grofo confirme : "Il a connu les Roms en premier à Paris. Il a appris le romanès, mais il ne voulait pas le parler, il comprenait tout. Mais il disait que parler le romanès comme un gadjo c'était pas la peine, les Roms se seraient moqués. Les Roms sont les plus blagueurs, les enfants l'auraient écouté et auraient ri, et nous aussi on aurait blagué. Il savait tout cela, alors il ne parlait pas, il était timide, discret, il ne posait pas de question, il écoutait, alors il savait tout. Nous on lui disait «tu es un espion », mais c'était un frère, il disait rien. Il savait nos secrets, la manière dont on vit, comment on fait les choses, la vie. Mais si tu vas en Afrique et au début tu regardes, tu t'étonnes et puis après c'est la vie, ce n'est plus des secrets, c'est juste comment on fait les choses, comment on mange, comment on dort, et tout. La vie normale, banale, alors il n'y a rien à dire, et Philippe n'a pas eu envie d'écrire la vie des Roms. Quand il allait dans un autre pays où les Roms ne parlaient pas français alors il parlait romanès, il savait bien parler, il comprenait tout mais avec nous il parlait français, nous les Roms français on parle bien le français aussi. Et il aidait certains à apprendre à lire. »

\section{Lick et le monde sinto}

Philippe de Marne ira régulièrement aux Saintes-Maries-de-la-Mer pour le pèlerinage du mois de mai. En 1961 il effectue une mission pour le compte du Musée des arts et traditions populaires et dépose dans le fonds du musée un enregistrement de cantiques français et provençaux (consultable à la phonothèque de la MMSH à Aix-en-Provence). Puis il y reviendra d'abord avec les Roms de Paris puis plus tard en 1967 avec Lick pour rejoindre la famille de celui-ci.

Que ce soit avec les Roms ou avec Lick l'amitié est là. Philippe recueille beaucoup d'informations («il remontait jusqu'à la septième génération » précise la femme de Grofo »). Il ne fait pas que cela puisqu'il apprend à lire à certains enfants roms et initie Lick à la littérature : «j'étais un peu autodidacte, j'avais à peine appris à lire et à écrire, j'étais allé à l'école quelques hivers ; de huit à douze ans je suis allé à l'école, et pas toute l'année, mon père et ma mère m'envoyaient à l'école pour les allocations ... et Philippe il m'a donné des livres, le premier c'était Saint Exupéry, Le Petit Prince » (Lick). Mais surtout pendant les années parisiennes de Lick ce sont des heures de conversation entre les deux jeunes gens même après que Philippe de Marne a démissionné du CNRS.

\section{Un entrepreneur hasardeux}


Il s'essaye au métier d'imprésario pour faire connaître Lick, finance ses disques « mais il suivait pas, les disques restaient dans les placards » et perd de l'argent : « il était anticommerçant, il se faisait avoir, c'était un grand couillon ... » En même temps « il travaillait beaucoup avec les petits papiers, avec la Bourse, il était très fort pour ça, coter en Bourse, moi j'y connais rien mais des fois il gagnait sa vie comme ça aussi ». Il montera un atelier de brochage en ayant acheté une usine dans le XIe arrondissement à Paris : «mais écoutait personne, il se croyait le grand organisateur, il se faisait avoir. L'atelier travaillait 24 heures sur 24, il y a même des Tsigane qui y ont travaillé. Mais il écoutait personne, il y avait un couple qui connaissait l'imprimerie, mais ils sont partis, je lui disais - tu vois Philippe tu es le plus grand organisateur, parce que on se loupait pas tous les deux, on était comme des frères ; j'abrège un petit peu ; l'affaire n'a pas marché mais il a quand même fait une affaire quand il a revendu l'usine, il l'avait achetée 37 millions de l'ancien temps et il l'a revendu 130 ! ». Pour autant Philippe de Marne n'a pas perdu la fibre ethnologique il fait connaissance du monde sinto aux Saintes en 1967 avec Lick qu'il suit au Plan de Grasse où vit toute sa famille. Il tente de faire des généalogies comme il l'a fait quelques années plus tôt avec les Roms de Paris. «On a essayé, on a fait les cimetières mais on n'a pas remonté haut parce que à l'époque chez les Tsiganes (Sinti) à l'époque là où ils s'arrêtaient ils les enterraient là, on a fait les cimetières et tout ça et puis il y avait pratiquement rien d'écrit, on n'est pas remonté très très loin. Et puis il me dit -il faut que tu écrives, mais moi je sais pas écrire, moi je suis auteur-compositeur, ça ça va mais je suis pas écrivain et lui sur les Roms il disait - moi, je les connais trop. Il ne voulait pas les dévoiler, par estime si tu veux. » Philippe essaye donc de convaincre Lick d'écrire, il tente même de proposer le projet à Jean Malaurie, directeur de la prestigieuse collection «Terre humaine », qui lui veut un texte de l'ethnologue, ce qui ne se fera pas mais Lick écrira finalement Scènes de la vie manouche. Sur les routes de Provence avec les Sinti Piémontais, 1935-1945.

\section{Un timide qui se replie sur son monde}

Grofo et Lick décrivent donc le même homme, extrêmement timide, réservé, mais aussi naïf et têtu, fidèle en amitié mais ne donnant pas signe de vie durant des années car pris par de nouveaux projets, de nouvelles tentatives. Il s'essayera à la pisciculture sur les terres familiales de Sologne, mais se lasse vite. Lick se souvient lui avoir rendu visite dans ces années là et fut frappé de voir des bulldozeurs abandonnés dans les fourrés, « il y en avait pour des millions, mais cela ne l'intéressait plus ». Quelques années plus tard Philippe de Marne se passionne pour le Cambodge et surtout les Cambodgiens ; mais : « il a dû perdre des millions là-bas ! C'était terrible ce gars-là ! Il avait fait une banque pour les paysans, pour leur prêter, et là encore il y a quelqu'un qui est venu pour le rouler et qui a tout emporté ; il s'est fait rouler d'un drôle de paquet et tout ça pour bien, mais quand tu es naïf, c'est ... »

Pour insister sur la timidité maladive de cet homme qui est allé en Amazonie, est devenu ami des Roms jusqu'à renoncer à son métier et à écrire quand il est convaincu qu'il les connaît trop et ne peut trahir leur confiance en publiant, Lick raconte une anecdote :

«Philippe était un homme très timide. Très respectueux des autres, extrêmement timide, et avec les femmes ne fallait pas en parler. Il ne s'est jamais marié, il a failli et je l'ai vu cette 
fille mais il était tellement orgueilleux il ne voulait pas qu'une femme le dépasse, qu'elle soit plus intelligente. Je l'avais vue, et il m'avait - elle a des problèmes aussi ... c'était une aristocrate aussi, ... (blanc) je te le dis quand même il y avait une fille extraordinaire qui était aristocrate aussi, elle s'appelait Inès, on s'est rencontré pour l'inauguration (sortie) du premier disque (enregistré par Lick et financé par de Marne) elle était ethnologue aussi, elle parlait sept langues, quand même, c'est ça qui lui faisait peur. J'ai vu qu'elle s'intéressait aussi à Philippe et je lui ai dit - c'est la femme qu'il te faut Philippe. Un soir ils sont allés dîner ensemble. Mais par sa grande timidité ... Je lui ai dit - Retéléphone à Inès vous êtes du même milieu je crois que cette fille c'est le bon moment. Des mois ont passé et je lui dis un jour tu vas téléphoner et elle te dira - tu vois je suis mariée, et c'est ce qui est arrivé. Voila. Mais à mon avis je crois qu'il n'y tenait pas tellement à être avec une femme car il était aussi très libre. C'était quand même un grand solitaire. Quand j'ai quitté Paris après on s'écrivait pratiquement pas. Sur ce plan-là c'était un peu terrible de pas avoir de nouvelles mais il se renfermait dans son monde. » 